\title{
A magic bullet for gout?
}

\author{
Alexander So, Nathalie Busso
}

Do we need new treatments for gout? Hyperuricaemia, the metabolic condition underlying gouty inflammation, has attracted recent attention as new uratelowering treatments emerge on the horizon. For an acute attack of gout, the currently available drugs-non-steroidal anti-inflammatory drugs, colchicine and corticosteroids, seem to do a pretty good job for the majority of patients in relieving the acute symptoms of inflammation. However, recent data have highlighted the epidemiological shifts in the gouty population towards a more elderly group of patients who often present multiple medical problems. This as well as clinical practice have made us aware that our current treatments of acute gout are not ideal in all situations. They may cause significant side effects in patients with poor renal and cardiac function, and practical experience indicates that quite a number of patients with chronic gout end up receiving long-term steroids, with all the side effects that such treatment engenders. Furthermore, there are abundant reports pointing out that gout is poorly managed in routine clinical practice, suggesting that we are not using all the tools at our disposition adequately. Any new treatment for gout should therefore offer distinct advantages over existing ones to justify its introduction.

With the discovery of the inflammasome as a major regulator of interleukin $1 \beta$ (IL1 $\beta$ ) processing and secretion in macrophages and dendritic cells, IL1 has once again returned to the spotlight as a mediator of acute inflammation. The identification of mutations in the NALP3 gene, a component of the inflammasome, in patients with a subset within the autoinflammatory syndromes (now called cryopyrin-associated periodic syndromes or CAPS) led to the idea of targeting IL1 $\beta$ in this disease. The results have been spectacular $^{12}$ and confirmed that IL1 inhibitors can have major clinical effects if the physiopathology of the disease involves excessive IL1 production. Martinon et al showed that monosodium urate (MSU)

Centre Hospitalier Universitaire Vaudois, Lausanne, Switzerland

Correspondence to: Professor A So, Department of Rheumatology, University Hospital Lausanne, 1011, Lausanne, Switzerland; alexanderkai-lik.so@chuv.ch crystals can directly trigger the inflammasome to process IL1 $\beta$, implying that acute gout may also be an IL1 $\beta$-mediated

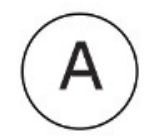

disease. $^{3}$ This concept has been strengthened by the findings of an open study on the effects of anakinra in gout ${ }^{4}$ and the articles by Torres and Terkeltaub in this issue of the journal (see articles on pages 1602 and 1613). ${ }^{5}{ }^{6}$ Torres showed convincingly in his animal studies that IL1 is a major trigger of joint inflammation. Using a new animal model of gout, where he injected MSU

Figure 1 Mode of action of interleukin 1 (IL1) and its inhibitors. (A) Binding of IL1 $\beta$ to the IL1 receptor type I (IL1RI) heterodimer complex results in signal transduction. IL1 $\beta$ binds to the membrane-bound IL1RI to form a complex with the IL1 receptor accessory protein (IL1RAcP). This complex recruits the IL1RI-associated kinase (IRAK), leading to signal transduction and gene activation. Receptor activation on cartilage and bone cells promotes cartilage breakdown and bone resorption within the joints. In the bone marrow, IL1 $\beta$ mobilises granulocyte progenitors and mature neutrophils, increases platelet production and augments lymphocyte responses to antigen and mitogens, leading to exacerbation of inflammatory responses. In the brain, IL1 $\beta$ triggers IL1 receptors in the hypothalamus, activating the centre for fever production via production of cyclooxygenase-2 and prostaglandin E2. IL1 also activates the endothelium to increase leukocyte adhesion and the production of IL6. (B) Anakinra is an antagonist of IL1 activity. Anakinra is a nonglycosylated recombinant human IL1 receptor antagonist (IL1Ra) that is produced in Escherichia coli. It binds to the IL1RI with similar affinity as IL1 but prevents IL1RAcP binding thus preventing signal transduction. Anakinra mimics the action of the endogenous IL1Ra, and inhibits both the membrane-bound IL1 and circulating IL1 isoforms. (C) Rilonacept inhibits IL1 activity. Rilonacept (also called IL1 Trap) is a recombinant protein comprising the extracellular domains of both IL1RI (red) and adaptor protein IL1RAcP (green) linked by the Fc region of the human IgG1 molecule (represented in brown). Rilonacept binds to IL $1 \alpha$, IL $1 \beta$ and IL $1 R a$ (with a lower affinity), thus preventing IL1 from binding to IL1RI. CRP, C-reactive protein; SAA, serum amyloid A. 


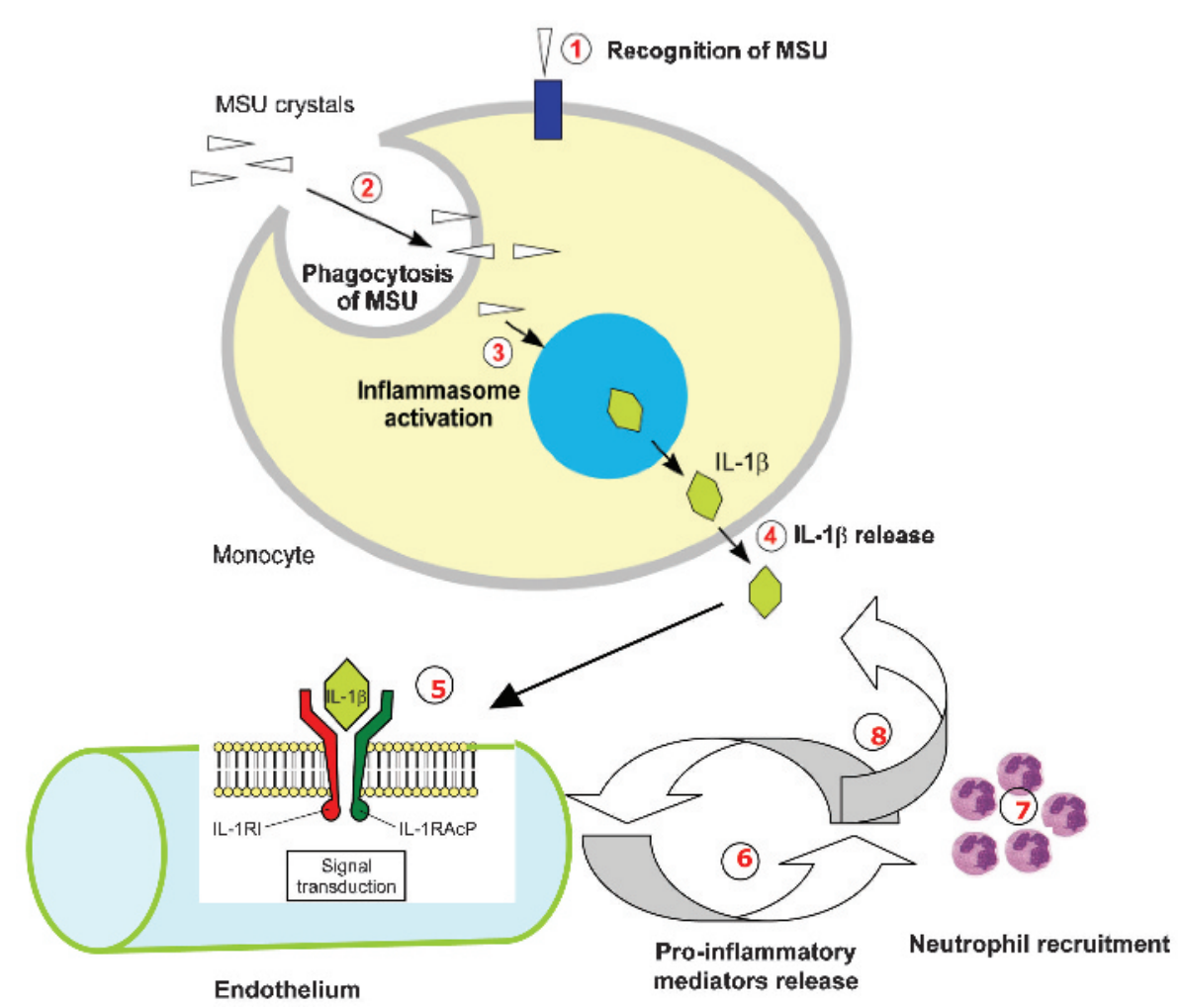

Figure 2 Multiple steps in the inflammatory cascade initiated by monosodium urate (MSU) crystals. (1) MSU crystals are detected by the pattern recognition molecules of the innate immune system, such as the Toll-like receptors. (2) MSU crystals are phagocytosed by macrophages. (3) Intracellular MSU crystals activate the NALP3 inflammasome, a multi-protein complex composed of the NALP3 sensor, the ASC adaptor protein and the procaspase-1. This induces cleavage of procaspase- 1 to its active form, caspase-1. Caspase- 1 in turn cleaves inactive pro-IL1 $\beta$ to generate IL1 $\beta$. (4) Active IL1 $\beta$ is then released into extracellular fluids (synovial fluid, plasma...). (5) IL1 $\beta$ activates IL1 receptors on the endothelium, resulting in signal transduction and gene activation. (6) An array of proinflammatory cytokines and chemokines, including IL8, is secreted by endothelial cells, which increase cell adhesion molecules on the endothelium. (7) Neutrophils are recruited leading to accumulation of these cells within the joint. (8) Amplification of the inflammatory cascade by neutrophil release of more IL1 $\beta$ and other proinflammatory cytokines.

crystals directly into the mouse ankle joint, he showed that signs and symptoms of acute inflammation were significantly reduced in the mice deficient for the IL1 receptor (IL1R) or when the action of IL1 was blocked by IL1 Trap (also called rilonacept), a molecule that has recently been licensed for the treatment of CAPS (fig 1). The novelty of his model was the measurement of nociception and mobility in mice, readouts that reflect the functional impairment caused by joint inflammation.

The same molecule, rilonacept, was tested in man by Terkeltaub and his colleagues in a small pilot study on 10 patients with chronic gout to validate the concept of IL1 inhibition for acute signs and symptoms of gout (fig 1). In comparison with the run-in period when patients received placebo, patients who received rilonacept weekly over 6 weeks showed a significant reduction in pain score and in C-reactive protein. The reduction in symptoms and signs were significant at exposed to MSU crystals was shown to be non-bone marrow-derived cells, as bone marrow chimeric mice (wild-type bone marrow transferred to IL1R knockout (KO) mice) did not mount an inflammatory response when challenged with an intraperitoneal injection of MSU crystals. These results showed that non-haemopoietic cells are critical for the expression of inflammation, and the most likely candidate is endothelial cells. ${ }^{8}$ Figure 2 shows the proposed model to explain how IL1 is critical to inflammation. A secondary question is whether IL1 $\beta$ alone accounts for inflammation, or does IL1 $\alpha$ also play a role? As anakinra and rilonacept block both forms of IL1, we do not have a clear answer from these results. A monoclonal antibody specific for IL1 $\beta$ is effective in the treatment of CAPS, and clinical studies are in progress in gout, so we will have a definitive answer in due course.

Apart from IL1, are other cytokines involved in acute gouty inflammation? From the results obtained in Torres' study, multiple proinflammatory cytokines and chemokines are upregulated at the same time as IL1 $\beta$, including tumour necrosis factor $\alpha$ (TNF $\alpha$ ), IL6, granulocyte-colony stimulating factor (G-CSF), KC (CXCL-1) and MCP-1 (CCL2). Interestingly, when the action of IL1 was blocked (in the IL1R KO or by rilonacept), upregulation of TNF, IL6 and G-CSF was also attenuated, but the production of the chemokines were less affected. These findings suggest a hierarchical order of cytokine production when MSU triggers inflammatory processes, some of which are IL1 dependent. To date, there are few clinical data to show that TNF $\alpha$ or IL6 blockade is clinically effective for gout, but it would be of interest to compare the effects of inhibiting different cytokines in vivo. From the available data, it appears that inhibiting IL1 alone in vivo can successfully turn off the inflammatory process, suggesting that IL1 $\beta$ release is indeed at the starting point of acute gout.

If indeed IL1 inhibition turns out to be an effective treatment, the questions that will be asked will include whether such an approach is warranted and what are the associated risks? The experience with IL1 inhibitors in clinical use (with agents such as anakinra and canakinumab) indicates that the treatment is generally safe, though there are always concerns about infectious complications when proinflammatory cytokines are inhibited. However, if inhibition is applied only in an acute setting, such complications should be less 
prominent than in chronic inhibition, as used when treating rheumatoid arthritis. If this treatment were to be applied to acute gout in the future, it would be vital that the target group of patients is clearly defined to justify its use over that of conventional agents, and that septic arthritis is excluded for obvious safety reasons.

Current research into the biochemical pathways that MSU crystals employ to trigger inflammation will open novel perspectives on anti-inflammatory treatment. Our understanding of the steps leading to the assembly of the inflammasome is still sketchy, but other molecules such as reactive oxygen species and purinergic receptors have been reported to be implicated. ${ }^{10}$ We should also not forget that MSU crystals can activate other inflammatory pathways, such as complement and mast cells. If we can put all this information together in a functional system, perhaps one day we will be able to select the target that is the most appropriate for a given patient to suppress the inflammatory process of acute gout.

Competing interests: None.

Provenance and peer review: Commissioned; externally peer reviewed.

Accepted 22 July 2009

Ann Rheum Dis 2009;68:1517-1519

doi:10.1136/ard.2009.112508

\section{REFERENCES}

1. Hawkins PN, Lachmann HJ, Aganna E, et al. Spectrum of clinical features in Muckle-Wells syndrome and response to anakinra. Arthritis Rheum 2004:50:607-12.

2. Lachmann HJ, Kone-Paut I, Kuemmerle-Deschner $\mathrm{JB}$, et al. Use of canakinumab in the cryopyrinassociated periodic syndrome. N Engl J Med 2009;360:2416-25.
3. Martinon F, Petrilli V, Mayor A, et al. Goutassociated uric acid crystals activate the NALP3 inflammasome. Nature 2006;440:237-41.

4. So A, Desmedt T, Revaz S, et al. A pilot study of IL-1 inhibition by anakinra in acute gout. Arthritis Res Ther 2007:9:R28.

5. Torres R, Macdonald L, Croll SD, et al. Hyperalgesia, synovitis, and multiple biomarkers of inflammation are suppressed by interleukin 1 inhibition in a novel animal model of gouty arthritis. Ann Rheum Dis 2009;68:1602-8.

6. Terkeltaub R, Sundy J, Schumacher HR, et al. The interleukin 1 inhibitor rilonacept in treatment of chronic gouty arthritis: results of a placebo-controlled, crossover pilot study. Ann Rheum Dis 2009:68:1613-17.

7. Dinarello CA. Immunological and inflammatory functions of the interleukin-1 family. Annu Rev Immunol 2009:27:519-50.

8. Chen CJ, Shi Y, Hearn A, et al. MyD88-dependent IL1 receptor signaling is essential for gouty inflammation stimulated by monosodium urate crystals. J Clin Invest 2006;116:2262-71.

9. Surprenant A, North RA. Signaling at purinergic P2X receptors. Annu Rev Physiol 2009; 71:333-59.

10. Martinon F, Mayor A, Tschopp J. The inflammasomes: guardians of the body. Annu Rev Immunol 2009;27:229-65.

\section{BMJ Careers online re-launches}

BMJ Careers online has re-launched to give you an even better online experience. You'll still find our online services such as jobs, courses and careers advice, but now they're even easier to navigate and quicker to find.

New features include:

- Job alerts - you tell us how often you want to hear from us with either daily or weekly alerts

- Refined keyword searching making it easier to find exactly what you want

- Contextual display - when you search for articles or courses we'll automatically display job adverts relevant to your search

- Recruiter logos linked directly to their organisation homepage - find out more about the company before you apply

- RSS feeds now even easier to set up

Visit careers.bmj.com to find out more. 